\title{
General analysis of instabilities and oscillations of the sequential tunneling in superlattices
}

\author{
X. R. Wang \\ Physics Department, The Hong Kong University of Science and Technology, Clear Water Bay, Hong Kong, China \\ Q. Niu \\ Physics Department, University of Texas, Austin, Texas 78712 \\ and Center for Theoretical Sciences, Hsinchu. Taiwan 300, Republic of China
}

(Received 22 February 1999)

\begin{abstract}
We present a general analysis of instabilities and oscillations of the sequential tunneling current in superlattices, based on the current-voltage characteristic of a single barrier. Our results depend only on the presence of a negative differential resistance region and are independent of the details of the model. We establish general conditions for the existence of a stable oscillatory region of current-voltage characteristic of the superlattice, and for instability leading to current self-oscillation. Our formalism permits a natural connection to standard results of chaos theory. [S0163-1829(99)50520-1]
\end{abstract}

Following the original suggestion of Esaki and co-workers, ${ }^{1,2}$ there has been a great deal of experimental ${ }^{3}$ and theoretical ${ }^{4}$ work on resonant tunneling in double barrier quantum well structures and superlattices. Under the right conditions, such structures have current-voltage characteristics with regions of negative differential resistance (NDR), leading to a range of important possible applications. Many interesting phenomena related to the NDR have been found in superlattices (SL), ranging from current-voltage oscillations on the sequential resonance tunneling plateau, ${ }^{5-10}$ current self-oscillations, ${ }^{11-13}$ and chaos. ${ }^{14}$ Self-oscillation has been observed in both doped and undoped SL systems. ${ }^{10-12}$ The oscillation can be induced by continuous illumination of a laser light ${ }^{13}$ or by change of doping. ${ }^{11}$ Recently, it has been shown that this self-oscillation can also be induced by applying an external magnetic field parallel to the SL layers. ${ }^{15}$

On the theoretical side, it is understood that the $I-V$ oscillation is related to the formation of stationary electric-field domains ${ }^{16}$ while the current self-oscillation is attributed to the motion of a domain boundary. ${ }^{17}$ Different theoretical methods include the continuum model approach, ${ }^{16}$ and the approach based on the rate equation for charge-carrier densities in quantum wells. ${ }^{17}$ By introducing a phenomenological carrier drift velocity curve, the second approach is capable of modeling both the formation of stationary electricfield domain and current self-oscillations. There are also microscopic Green's function calculations. ${ }^{18}$ While the microscopic approach would be accurate if all the microscopic parameters and mechanisms were known, it remains a challenge to deduce the rules of macroscopic behavior from the microscopic details. In this paper, we present a general analysis of instabilities and oscillations due to the existence of NDR in superlattices, under very general conditions. We make no assumption about the origin of the NDR, and we dispense with the concept of drift velocity, which is not well defined in a tunneling situation. We take the single well $I-V$ characteristic, containing NDR regions, as given rather than starting from a first-principles microscopic model. This NDR may be due to any microscopic mechanism, such as offresonance tunneling, ${ }^{19}$ or the Gunn effect. ${ }^{20}$ Our goal is to study the consequences of this NDR in a superlattice. We will show that stationary electric-field domains, current selfoscillations, and chaos are direct consequences of the NDR under certain conditions. In this sense, our analysis might be applicable to other systems.

We consider a SL system consisting of $N$ quantum wells as shown schematically in Fig. 1. An external bias $U$ is applied between the two end wells. Current flows perpendicular to the SL layers. We make the following basic assumptions throughout our analysis. (1) Inside each well, the electronic states are described fully by time-independent quantum mechanics. This implies that we assume that the well width is smaller than the coherence length. (2) Charge carriers are in local equilibrium within each well, so that a chemical potential can be defined locally. The chemical potential difference between two adjacent wells is called the bias $V$ between the two wells. (3) For a given bias $V$ between two adjacent wells, a current $I(V)$ passes through the barrier between them. Different wells may have different $I(V)$ functions. Doping and external perturbations such as a magnetic field can modify $I(V)$ by changing the electron states involved in transport. Under these assumptions, we obtain two sets of $N$ equations. Charge conservation gives

$$
\frac{\partial n_{i}}{\partial t}=I_{i-1}\left(\mu_{i-1}-\mu_{i}\right)-I_{i}\left(\mu_{i}-\mu_{i+1}\right)
$$

while the discrete Poisson equation gives

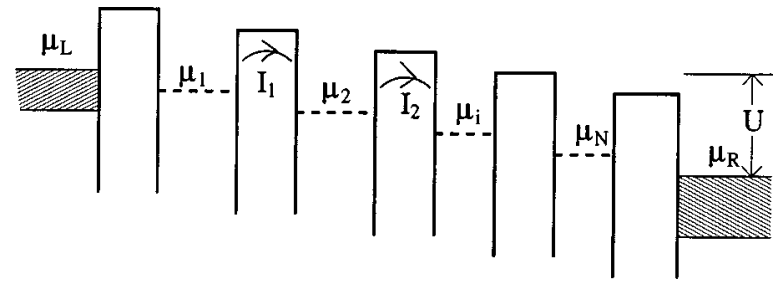

FIG. 1. Schematic illustration of an SL system. $\mu_{i}$ is the local chemical potential of the $i$ th well. $\mu_{L}$ and $\mu_{R}$ are the chemical potential of left-hand side and right-hand side electrodes, respectively. $\mu_{L}-\mu_{R}=U$ is the external bias. 


$$
\mu_{i-1}-2 \mu_{i}+\mu_{i+1}=k n_{i}
$$

Here $i=1,2, \ldots, N . n_{i}$ and $\mu_{i}$ are the excess charge density and the chemical potential of $i$ th well, respectively. $I_{i}\left(V_{i}\right)$ is the tunneling current through the barrier separating $i$ th and $(i+1)$ th wells under bias $V_{i} \equiv \mu_{i}-\mu_{i+1} . k$ is a constant $4 \pi l^{2} / \epsilon$ where $\epsilon$ and $l$ are the permittivity and periodicity of the SL, respectively. Furthermore, $\mu_{0}=\mu_{L}$ and $\mu_{N+1}=\mu_{R}$ are the chemical potentials of left-hand side and right-hand side electrodes. Under an external dc bias $U$, there is an extra constraint

$$
\mu_{L}-\mu_{R}=U
$$

Steady-state solutions (SSS) are determined by the following set of $N+1$ equations:

$$
\begin{gathered}
I_{i-1}\left(\mu_{i-1}-\mu_{i}\right)-I_{i}\left(\mu_{i}-\mu_{i+1}\right)=0 \\
\mu_{0}-\mu_{N+1}=U .
\end{gathered}
$$

The strategy of our analysis is as follows. We will first analyze the stability of a SSS in order to find conditions for instability. We then show that current self-oscillations must occur if all steady-state solutions are unstable. Then we show that a SL system has unstable solutions only under certain conditions. Let $\left\{\mu_{i}\right\}=\left\{\mu_{i 0}\right\}$ be a SSS. We linearize the equations around the SSS. Denote $V_{i}=\mu_{i}-\mu_{i+1}, V_{i}^{0}=\mu_{i-10}$ $-\mu_{i 0}$, and $E_{i}=V_{i}-V_{i}^{0}$, we obtain the following set of equations for the $E_{i}$ :

$$
\begin{gathered}
\dot{E}_{i-1}+k I_{i-1}^{\prime}\left(V_{i-1}^{0}\right) E_{i-1}=\dot{E}_{i}+k I_{i}^{\prime}\left(V_{i}^{0}\right) E_{i} \\
\sum_{i=0}^{N} E_{i}=0,
\end{gathered}
$$

where $\dot{E}_{i}$ is the time derivative of $E_{i}$, and $I_{i}^{\prime}\left(V_{i}^{0}\right)$ is the derivative of $I_{i}$ with respect to the potential difference $V_{i}$ at $V_{i}^{0}$. Putting $E_{i}=A_{i} e^{-\lambda t}$, the steady-state solution will be stable if and only if all possible $\lambda$ are positive. Otherwise, the steady solution $\mu_{i}=\mu_{i 0}$ is unstable. It is easy to show that $\lambda$ satisfies the following secular equation: ${ }^{21}$

$$
\prod_{j=0}^{N}\left(\lambda-\alpha_{j}\right) \sum_{i=0}^{N} \frac{1}{\lambda-\alpha_{i}}=0,
$$

where $\alpha_{i}=k I_{i}^{\prime}\left(V_{i}^{0}\right)$ is proportional to the differential conductance of the $i$ th well at bias $V_{i}^{0}$. This is a $N$ th order algebraic equation with $N$ roots. Let us order the $\alpha$ 's, $\alpha_{0} \leqslant \alpha_{1} \leqslant \alpha_{2} \cdots \leqslant \alpha_{N}$. Then one can show that Eq. (6) has one and only one root between $\left[\alpha_{j}, \alpha_{j+1}\right] .{ }^{22}$ Therefore, we can make three statements about the SSS $\left(\left\{\mu_{i}\right\}=\left\{\mu_{i 0}\right\}\right)$. (1) Equation (6) has only positive roots when $\alpha_{i}>0$ for all $i$, i.e., every well is in the positive differential resistance (PDR) region. Therefore, the SSS is stable in this case. (2) The SSS must be unstable if two or more wells are in the NDR region, i.e., $\alpha_{0}<0$ and $\alpha_{1}<0 . \alpha_{i}>0$ for $i \geqslant 2$. This statement is not trivial. (3) There are two possibilities when one of the wells is in the NDR region while the rest of the wells are in the PDR region, i.e., $\alpha_{0}<0$ and $\alpha_{i}>0$ for the rest of $i$. In the small NDR case the steady-state solution is stable if the NDR is smaller than the sum of all PDR, i.e., $-1 / \alpha_{0}$ $<\sum_{i=1}^{N} 1 / \alpha_{i}$. In the large NDR case the solution is unstable

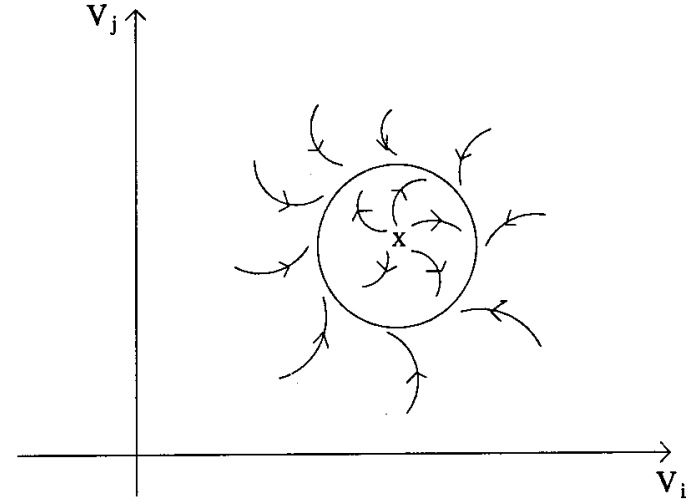

FIG. 2. Schematic illustration of the flow of the phase point of a SL system around an unstable fixed point in the phase space of biases on the wells. The arrows indicate the direction in which the system is driven. The closed curve is called a limit cycle and corresponds to periodic motion of the system.

when the above condition is violated, i.e., the magnitude of the NDR is larger than the sum of the PDR. These statements simply restate the well-known fact that a circuit is stable if the series dissipative resistance is larger than the negative differential resistance. So far, our statements are general. $I(V)$ can be different for different wells, and depends, in general, on the material parameters, well structure, doping concentration, and other external fields.

To proceed with our analysis, we consider the phase space of the biases of each well, as schematically shown in Fig. 2, where for illustration, we consider the space to be a plane. A SSS is a point in this space: in mapping language, it is a fixed point of our system, which may be stable or unstable. The question is, what is the behavior of a system when its only fixed point is unstable? Assume that the point labeled by a cross in Fig. 2 is the unstable fixed point of the system. A small deviation from this point will produce drastic consequences, the direction of all flow being outwards from this point. However, because the potential difference between two adjacent wells cannot exceed the applied bias, an unstable fixed point must be attractive in regions far away from the fixed point. Local repulsion and global attraction of the flow requires the formation of a closed curve around the unstable fixed point. The physics is exactly the same as in chaos theory where this closed curve is called a limit cycle. ${ }^{23}$ The process of generating a limit cycle from a fixed point is often called a Hopf bifurcation. In this simple case, it corresponds to a periodic motion of the system. More complicated behavior can occur when we do not limit the phase space to be a plane. We shall come back to this point later.

The next natural question is, under what conditions does our SL system have only unstable SSS? In order to show that this is indeed possible, and to establish these conditions, we will consider a highly idealized model of $N-1$ wells separated by $N$ barriers similar to Fig. 1 . We will assume that the tunneling current between any two neighboring wells is described by the same piecewise linear $I(V)$ function (Fig. 3)

$$
I(V)=\left\{\begin{array}{cc}
\alpha V, & V \leqslant F_{1} \\
\alpha F_{1}-\beta\left(V-F_{1}\right), & F_{1}<V<F_{2} \\
\alpha(V-\Delta F), & V \geqslant F_{2} .
\end{array}\right.
$$




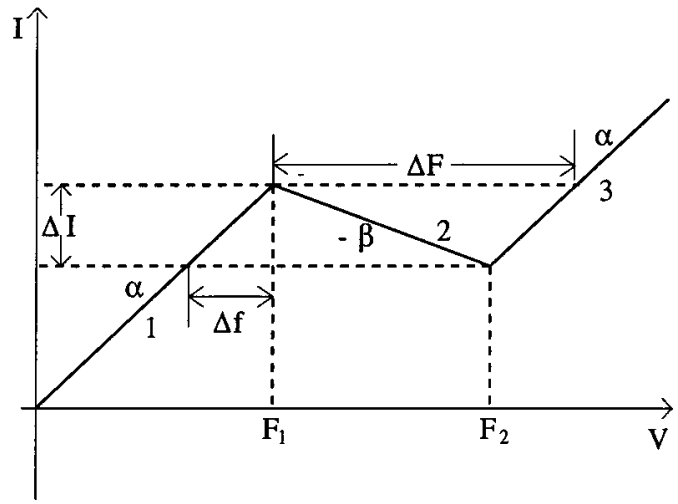

FIG. 3. Piecewise linear $I(V)$ curve where $V$ is the bias across one barrier. Lines 1 and 3 have the same slope $\alpha$. Line 2 has the negative differential conductance $-\beta(\mathrm{NDR}=-1 / \beta)$.

For simplicity, we assume lines 1 and 3 have the same slope, $\alpha$. The $I(V)$ curve has a negative differential conductance (resistance) region between $F_{1}$ and $F_{2}$ (line 2). The negative differential conductance is assumed to have a constant value $-\beta$. The meanings of $\Delta F, \Delta f$, and $\Delta I$ are given in Fig. 3 . Substituting this $I(V)$ curve into Eq. (4), we find the following results. For $U \leqslant N F_{1}$ or $U \geqslant N\left(F_{1}+\Delta F\right)$ there is a stable SSS with the same potential difference $V_{i}=U / N$ across each barrier. $I=\alpha U / N$ in the first case and $I=\alpha(U-N \Delta F) / N$ in the second.

For the intermediate region of $U, N F_{1}<U<N F_{1}+\Delta F$, we have to consider the cases $\Delta F / \Delta f<N$ (i.e., $1 / \beta<(N$ $-1) / \alpha$ : the "small NDR'" case) and $\Delta F / \Delta f>N$ (i.e., $1 / \beta$ $>(N-1) / \alpha$ : the "large NDR" case) separately, detailed as follows.

(a) $\Delta F / \Delta f<N$ (small NDR). Suppose that $0<U-N F_{1}$ $<\Delta F$. Then there is a stable SSS with all barriers in a PDR region of Fig. 3: one barrier has $V=(U-\Delta F) / N+\Delta F$ (i.e., it is in the high $V$ region of its characteristic) while all the others have $V=(U-\Delta F) / N$ and are in the low $V$ region. The current $I=\alpha(U-\Delta F) / N$. Similarly, if $0<U-N F_{1}$ $-k \Delta F<\Delta F$, where $0 \leqslant k \leqslant N-1$, there is a stable SSS with $(k+1)$ barriers in the high $V$ region and the remainder in the low $V$ region. The current is $I=\alpha[U-(k+1) \Delta F] / N$. Thus the $I(U)$ characteristic of the SL in this range of $U$ has the well-known sawtooth form with $N$ oscillations. ${ }^{10,15}$ No selfoscillation can occur.

(b) $\Delta F / \Delta f>N$ (large NDR). Now, we find that a stable SSS is only possible if $\Delta F-N \Delta f<U-N F_{1}-k \Delta F<\Delta F$. If $0<U-N F_{1}-k \Delta F<\Delta F-N \Delta f$, a SSS has at least one barrier in the NDR region, and our previous analysis has shown that such a solution is unstable. The system goes through a limit cycle process which may be periodic or chaotic.

While our analysis is based on a simplified model of the $I(V)$ characteristic of a single barrier, we believe that our conclusion is quite general that small, but positive and nonzero, negative differential conductance (i.e., large but finite NDR) is conducive to self-oscillation. This result explains why it is generally found experimentally that self-oscillation occurs when $\Delta I$, the peak to valley difference of the $I(V)$ characteristic (see Fig. 3), becomes small. ${ }^{15}$ How this small $\Delta I$ is achieved - whether by illumination, doping, magnetic field, or otherwise - is apparently irrelevant. Of course, detailed calculations are required in order to understand how a particular factor changes the $I(V)$ curve of a well.

It is worthwhile pointing out that our analysis does not depend on the microscopic mechanism of the NDR. Because of the generality of the analysis, the results might also be applicable to other systems such as electronic circuits containing NDR components. In the framework of our general analysis, both $I(V)$ oscillation and current self-oscillation can be attributed to the NDR. The facts that voltage drop occurs across a barrier and that the number of barriers in a SL must be an integer lead to only unstable SSS under certain conditions. Thus our analysis shows that the system has to undergo self-oscillation or, in principle, a possibly more complicated motion if the dimensionality of the unstable parameter space is larger than $3 .^{23}$

Before we end this paper, we would like to discuss other possible transport behavior of a SL. We mentioned that the limit cycle, and thus the periodic motion, is the outcome of competition between local repulsion and global attraction of the flow near an unstable fixed point in phase space. As is well known in chaos theory ${ }^{23}$ that another bifurcation can occur when the limit cycle loses stability and becomes repulsive locally. An attracting closed tube, a 2 torus, forms around the unstable limit cycle. This process might continue, leading to the so-called Landau-Hopf ${ }^{23}$ route to chaos. It would be extremely interesting to investigate whether one can realize the Landau-Hopf route in a SL system.

In summary, we present a general analysis of stability and oscillations of sequential tunneling in superlattices based on the current-voltage characteristic of a single well. The system is stable if the bias on all the individual wells are in the positive differential resistance (PDR) region. The tunneling current is stable when the bias of one and only one of the wells is in the negative differential resistance (NDR) region while the bias of the rest of the wells is in the positive differential resistance (PDR) region, and if the NDR is smaller than the sum of the values of all the PDR; otherwise it is unstable. The current is unstable if the biases of more than one well is in the NDR region. The system can undergo a limit cycle, and show oscillation in time even under a steady voltage. We also show, from an explicit example, that a SL can indeed become unstable when the negative differential conductance region becomes almost flat.

One of us (X.R.W.) would like to thank Professor P. Sheng for drawing his attention to this problem and for much valuable advice. He also wants to thank Professor M. Sturge for many useful suggestions and comments, and for a critical reading of the manuscript. He would also like to thank the hospitality of the Center for Theoretical Sciences, Taiwan. The authors would like to acknowledge Professor B. L. Hao and Professor C. S. Ting for stimulating discussions, and Dr. J. N. Wang and Dr. B. Q. Sun for communicating unpublished work. This work was supported by UGC, Hong Kong, through an RGC/DAG grant. 
${ }^{1}$ R. Tsu and L. Esaki, Appl. Phys. Lett. 22, 562 (1973).

${ }^{2}$ L.L. Chang, L. Esaki, and R. Tsu, Appl. Phys. Lett. 24, 593 (1974); L. Esaki and L.L. Chang, Phys. Rev. Lett. 33, 495 (1974).

${ }^{3}$ F. Capasso, Physics of Quantum Electron Devices (SpringerVerlag, New York, 1990); F. Capasso, K. Mohammed, and A.Y. Cho, Appl. Phys. Lett. 48, 478 (1986).

${ }^{4}$ J.N. Schulman, J. Appl. Phys. 60, 3954 (1986); T.B. Boykin, J.P. van der Wagt, and J.S. Harris, Jr., Phys. Rev. B 43, 4777 (1991); T. Weil and B. Vinter, Appl. Phys. Lett. 50, 1281 (1987); M.C. Payne, J. Phys. C 19, 1145 (1986).

${ }^{5}$ Semiconductor Superlattices: Growth and Electronic Properties, edited by H.T. Grahn (World Scientific, Singapore, 1995).

${ }^{6}$ Y. Kawamura, K. Wakita, H. Asaki, and K. Kurumada, Jpn. J. Appl. Phys., Part 2 25, L928 (1986); Y. Kawamura, K. Wakita, and K. Oe, ibid. 26, L1603 (1987).

${ }^{7}$ T. Furuta, K. Hirakawa, J. Yoshino, and H. Sakaki, Jpn. J. Appl. Phys., Part 2 25, L151 (1986); K. Hirakawa, Y. Shimada, and T. Ikoma, Physica B 227, 202 (1996).

${ }^{8}$ K.K. Choi, B.F. Levine, C.G. Bethea, J. Walker, and R.J. Malik, Appl. Phys. Lett. 50, 1814 (1987); K.K. Choi, B.F. Levine, N. Jarosik, J. Walker, and R.J. Malik, Phys. Rev. B 38, 12362 (1988); Y. Zhang, Y. Li, D. Jiang, X. Yang, and P. Zhang, Appl. Phys. Lett. 64, 3416 (1994).

${ }^{9}$ P. Helgesen, T.G. Finstad, and K. Johannessen, J. Appl. Phys. 69, 2689 (1991).

${ }^{10}$ S.H. Kwok, R. Merlin, H.T. Grahn, and K. Ploog, Phys. Rev. B 50, 2007 (1994); H.T. Grahn, R.J. Haug, W. Müller, and K. Ploog, Phys. Rev. Lett. 67, 1618 (1991).

${ }^{11}$ H.T. Grahn, J. Kastrup, K. Ploog, L.L. Bonilla, J. Galan, M. Kindelan, and M. Moscoso, Jpn. J. Appl. Phys., Part 1 34, 4526
(1995); J. Kastrup, R. Klann, H.T. Grahn, K. Ploog, L.L. Bonilla, J. Galan, M. Kindelan, M. Moscoso, and R. Merlin, Phys. Rev. B 52, 13761 (1995).

${ }^{12}$ H. Mimura, M. Hosoda, N. Ohtani, K. Tominaga, K. Fujita, T. Watanabe, H.T. Grahn, and K. Fujiwara, Phys. Rev. B 54, R2323 (1996); M. Hosoda, H. Mimura, N. Ohtani, K. Tominaga, T. Watanabe, K. Fujiwara, and H.T. Grahn, Appl. Phys. Lett. 69, 500 (1996).

${ }^{13}$ S.H. Kwok, T.B. Norris, L.L. Bonilla, J. Galan, J.A. Cuesta, F.C. Martinez, J.M. Molera, H.T. Grahn, K. Ploog, and R. Merlin, Phys. Rev. B 51, 10171 (1995).

${ }^{14}$ Y. Zhang, J. Kastrup, R. Klann, K. Ploog, and H.T. Grahn, Phys. Rev. Lett. 77, 3001 (1996).

${ }^{15}$ B. Q. Sun et al. (unpublished).

${ }^{16}$ B. Laikhtman, Phys. Rev. B 44, 11260 (1991); B. Laikhtman and D. Miller, ibid. 48, 5395 (1993).

${ }^{17}$ F. Prengel, A. Wacker, and E. Schöll, Phys. Rev. B 50, 1705 (1994); L.L. Bonilla, J. Galan, J.A. Cuesta, F.C. Martinez, and J.M. Molera, ibid. 50, 8644 (1994); A. Wacker, M. Moscoso, M. Kindelan, and L.L. Bonilla, ibid. 55, 2466 (1997).

${ }^{18}$ A. Wacker and A.P. Jauho, Phys. Rev. Lett. 80, 369 (1998); Phys. Scr. T69, 321 (1997).

${ }^{19}$ R.F. Kazarinov and R.A. Suris, Fiz. Tekh. Poluprovodn. 5, 797 (1971) [Sov. Phys. Semicond. 5, 707 (1971)]; Z.K. Tang and X.R. Wang, Appl. Phys. Lett. 68, 3449 (1996).

${ }^{20}$ J.B. Gunn, IBM J. Res. Dev. 8, 141 (1964).

${ }^{21}$ J.H. Wilkinson, The Algebraic Eigenvalue Problem (Clarendon Press, Oxford, 1988).

${ }^{22}$ E.T. Whittaker and G.N. Watson, A Course of Modern Analysis, 4th ed. (Cambridge University Press, Cambridge, 1990).

${ }^{23}$ Bai-Lin Hao, Chaos II (World Scientific, Singapore, 1990). 